\title{
Phase transformations induced by spherical indentation in ion-implanted amorphous silicon
}

\author{
B. Haberl, J. E. Bradby, ${ }^{\text {a) }}$ S. Ruffell, and J. S. Williams \\ Department of Electronic Materials Engineering, Research School of Physical Sciences and Engineering, \\ The Australian National University, Canberra, Australian Capital Territory 0200, Australia \\ P. Munroe \\ Electron Microscope Unit, University of New South Wales, Sydney, New South Wales 2052, Australia
}

(Received 14 November 2005; accepted 3 May 2006; published online 12 July 2006)

\begin{abstract}
The deformation behavior of ion-implanted (unrelaxed) and annealed ion-implanted (relaxed) amorphous silicon $(a-\mathrm{Si})$ under spherical indentation at room temperature has been investigated. It has been found that the mode of deformation depends critically on both the preparation of the amorphous film and the scale of the mechanical deformation. Ex situ measurements, such as Raman microspectroscopy and cross-sectional transmission electron microscopy, as well as in situ electrical measurements reveal the occurrence of phase transformations in all relaxed $a$-Si films. The preferred deformation mode of unrelaxed $a-\mathrm{Si}$ is plastic flow, only under certain high load conditions can this state of $a$-Si be forced to transform. In situ electrical measurements have revealed more detail of the transformation process during both loading and unloading. We have used ELASTICA simulations to obtain estimates of the depth of the metallic phase as a function of load, and good agreement is found with the experiment. On unloading, a clear change in electrical conductivity is observed to correlate with a "pop-out" event on load versus penetration curves. (c) 2006 American Institute of Physics. [DOI: 10.1063/1.2210767]
\end{abstract}

\section{INTRODUCTION}

Amorphous silicon $(a-\mathrm{Si})$ is both a technologically significant and physically interesting material with applications, for example, in photovoltaic cells ${ }^{1}$ and microelectromechanical systems. ${ }^{2}$ Since crystalline silicon $(c-\mathrm{Si})$ and $a$-Si are both tetrahedrally coordinated, they share many properties, such as mean bond lengths and mean bond angles. However, a number of properties differ between them, such as their respective electrical and mechanical properties. ${ }^{2,3}$

Crystalline $\mathrm{Si}$ is well known to undergo a series of phase transformations during mechanical deformation. ${ }^{4-7}$ Both high-pressure diamond anvil ${ }^{4,8-10}$ and indentation experiments ${ }^{5-7,11}$ have shown that crystalline diamond-cubic Si-I undergoes a $22 \%$ increase in density, which is due to a phase transformation during loading at a pressure of $\sim 11 \mathrm{GPa}$ to a metallic $\beta$-Sn (Si-II) phase. ${ }^{4,10} \mathrm{As} \mathrm{Si}-\mathrm{II}$ is unstable at pressures below $8 \mathrm{GPa},{ }^{4,8}$ the material undergoes further transformation on pressure release. Diamond anvil experiments have indicated the formation of the metastable polycrystalline phases Si-III (bc8) and Si-XII (r8) on pressure release. ${ }^{8,9}$ For indentation experiments, the final structure of the transformed volume depends critically on the unloading conditions, with slow unloading leading to the formation of the two polycrystalline phases (Si-III and Si-XII) but fast unloading ( $\geqslant \sim 50 \mathrm{mN} / \mathrm{s})$ leading to the formation of an amorphous silicon phase. ${ }^{11-13}$

An early high-pressure (diamond anvil) study on vacuum-evaporated $a$-Si reported a transformation to a metallic phase that was consistent with high density amorphous

${ }^{a)}$ Electronic mail: jodie.bradby@anu.edu.au
$\mathrm{Si}^{14}$ The remaining phase after unloading was (low density) $a$-Si. A further study based on diamond anvil results has also proposed that $a$-Si should undergo a transformation to a high density (metallic) amorphous phase during loading and transform back to its original low density $a$-Si state during unloading. ${ }^{15}$ Only one report ${ }^{10}$ of diamond anvil experiments on $a$-Si provides evidence for a transformation to a crystalline (Si-III) phase on pressure release. Indentation experiments on $a$-Si prepared by homogeneous chemical vapor deposition (unrelaxed) also indicated transformation to an electrically conducting phase ${ }^{16}$ with the remaining phase after unloading being (low density) $a$-Si. However, more recent indentation work on both unannealed (unrelaxed) and annealed (relaxed) ion-implanted $a-\mathrm{Si}$ is not consistent with these latter findings. ${ }^{17,18}$ For example, it has been shown that the relaxed state undergoes a phase transformation to a metallic phase under loading and can undergo further phase transformations to the polycrystalline phases Si-III and SiXII during unloading, whereas no evidence of phase transformations was found in the unrelaxed state of $a$-Si under low load indentation. We have previously ${ }^{18}$ speculated on possible reasons for the large difference in deformation behavior between relaxed and unrelaxed $a$-Si films and therefore will not discuss this aspect here in detail. We believe that the mechanical behavior can be explained by well documented structural differences ${ }^{19-22}$ between highly defective or paracrystalline unrelaxed $a$-Si and the fully fourfold coordination of relaxed $a$-Si which has only short range order and is a continuous random network (CRN).

In this current work, we focus on the behavior of ionimplanted $a$-Si under spherical indentation to gain more insight into the transformation pathways during indentation 
TABLE I. Ion-implantation energies and resulting $a$-Si thickness as measured using channeling Rutherford backscattering spectrometry.

\begin{tabular}{cc}
\hline \hline $\begin{array}{c}\text { Implantation energy } \\
(\mathrm{keV})\end{array}$ & $\begin{array}{c}\text { Thickness of } a-\mathrm{Si} \\
(\mathrm{nm})\end{array}$ \\
\hline 600 and 90 & 920 \\
400 and 90 & 650 \\
150 and 50 & 350 \\
40 & 100 \\
\hline \hline
\end{tabular}

loading and unloading. The deformation and transformation behavior of $a$-Si is compared with $c$-Si whose behavior has been extensively studied. ${ }^{5,6,11,13}$ This study employs ex situ techniques such as Raman microspectroscopy and crosssectional transmission electron microscopy (XTEM) as well as in situ electrical measurements. ${ }^{7}$

\section{EXPERIMENT}

Continuous surface amorphous layers of different thicknesses were created by the implantation of Si ions of different energies (see Table I) into single crystal $\mathrm{Cz} \mathrm{Si}(100), p$ doped for a resistivity of 5-10 $\Omega \mathrm{cm}$, at liquid nitrogen temperature using the ANU 1.7 MV NEC tandem high energy ion implanter. The fluence for each implantation was $\sim 3$ $\times 10^{15} \mathrm{~cm}^{-2}$. Thereafter, portions of the as-implanted $a-\mathrm{Si}$ samples were furnace annealed for $30 \mathrm{~min}$ at a temperature of $450{ }^{\circ} \mathrm{C}$ in an argon atmosphere. This was done to fully relax but not recrystallize the amorphous silicon layer. ${ }^{19,20}$ It is well established ${ }^{23}$ that the epitaxial growth of low-doped $a$-Si from the amorphous phase is negligible under these conditions. The resulting thickness of the films was measured by channeling Rutherford backscattering spectrometry using $2 \mathrm{MeV}$ helium ions with the results summarized in Table I.

A series of indentations was performed on unrelaxed and relaxed $a$-Si samples, as well as on $c$-Si samples for comparison, using an ultra-micro-indentation-system 2000 with spherical diamond indenters of $\sim 2.0$ and $\sim 5.0 \mu \mathrm{m}$ radii at room temperature and ambient pressure. Loads of up to $40 \mathrm{mN}$ were applied using the $\sim 2.0 \mu \mathrm{m}$ indenter and up to $80 \mathrm{mN}$ using the $\sim 5.0 \mu \mathrm{m}$ indenter at loading and unloading rates of $1-2 \mathrm{mN} / \mathrm{s}$ using a continuous load-unload cycle. These maximum loads result in similar stress levels under the indenters and hence in a similar maximum penetration depth.

After indentation, Raman spectra were recorded with a Renishaw 2000 Raman imaging microscope using the $632.8 \mathrm{~nm}$ excitation line of a helium-neon laser focused to a spot of $\sim 1.0 \mu \mathrm{m}$ radius.

To further investigate the deformed regions beneath the surface, XTEM samples were prepared from selected indents as described previously. ${ }^{11,13}$ These samples were first mechanically polished to $\sim 60 \mu \mathrm{m}$ thickness and finally milled to electron transparency using a FEIxP200 focused ion beam (FIB) system with a rastered beam of $30 \mathrm{keV}$ gallium ions. To shield the surface from excessive implantation induced damage during the ion milling process, a $1 \mu \mathrm{m}$ platinum layer was deposited on the surface using the FIB system. A

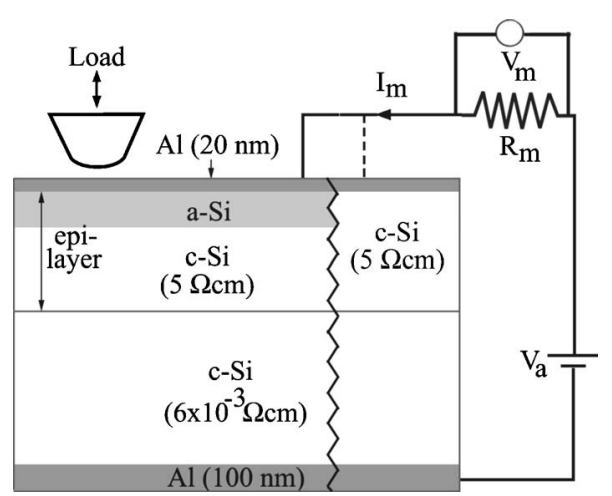

FIG. 1. Schematic of the experimental setup used for the electrical measurements. The $c$-Si sample consists of a 2 or $7 \mu \mathrm{m}$ epilayer Si grown on bulk $\mathrm{Si}$ with a low resistance, whereas for the $a$-Si sample a continuous surface amorphous layer was created in this high resistance Si epilayer. Al was evaporated on both sides of the samples and a circuit was connected across the sample (in the case of the $c$-Si sample the circuit is represented by the dotted line).

Philips CM 300 operating at an accelerating voltage of $300 \mathrm{kV}$ was used for the electron microscopy.

In situ electrical measurements were also carried out during indentation. ${ }^{7}$ For such measurements, 2 or $7 \mu \mathrm{m}$ epilayers of $p$-type boron doped $\mathrm{Si}(100)$ of $5 \Omega \mathrm{cm}$ resistivity grown on ( $p$-type) substrates of low resistivity (6 $\times 10^{-3} \Omega \mathrm{cm}$ ) were used. As described earlier, surface amorphous layers were created in the epilayer and portions of those samples were annealed. Aluminum layers were thermally evaporated onto the front $(\sim 20 \mathrm{~nm})$ and back surfaces $(\sim 100 \mathrm{~nm})$ of the samples. In the case of $c$-Si, a Schottky contact was formed at the Al-epilayer interface whereas, in the case of $a-\mathrm{Si}$, the $\mathrm{Al}$ was in contact with the (less conducting) $a$-Si. Due to the much higher carrier concentration in the substrate the back contact was Ohmic. As shown in Fig. 1 the sample was placed in series with a $1 \mathrm{k} \Omega$ resistor $\left(R_{m}\right)$ with a dc voltage of $1 \mathrm{~V}\left(V_{a}\right)$ applied across the circuit. In the case of $c$-Si, the applied voltage places the Schottky contact under reverse bias resulting only in flow of leakage current. Significant current will only flow if the Schottky contact is converted to an Ohmic contact. This occurs when the material directly under the contact is transformed to the metallic Si-II (or a metallic amorphous) phase at high pressures. An increase in current will cause a corresponding increase in the magnitude of $V_{m}$ (measured across the $1 \mathrm{k} \Omega$ resistor) and hence the total circuit current $I_{m}$ can be monitored as a function of time by measuring $V_{m}$ during indentation. In the case of the $a-\mathrm{Si}$, only leakage current can flow in the circuit until the transformed zone (the metallic phase) reaches the underlying $\mathrm{Si}-\mathrm{I}$. Once the entire thickness of $a$-Si is converted to the metallic phase under the indenter, current can flow through the low resistance path to the underlying $c$-Si. Hence our measurements also reveal the depth at which the transformed metallic phase connects to the underlying $\mathrm{Si}$-I.

\section{RESULTS}

\section{A. Thick a-Si films}

In this article an amorphous film is described as "thick" if it is $\geqslant 650 \mathrm{~nm}$. For these cases, under most indentation 



FIG. 2. Load-unload curves (with insets of the derivative of the load) of indentation into (a) unrelaxed and (b) relaxed $\sim 920 \mathrm{~nm}$ thick $a$-Si. The indentation was performed using an $\sim 5.0 \mu \mathrm{m}$ indenter tip. In the case of relaxed $a$-Si the "pop-out" event is clearly visible and the "pop-in" event is visible by a change in slope in the derivative.

conditions in this study, the tip penetrates less than half the film thickness and the deformation behavior of the $a$-Si film is not significantly influenced by the underlying $c$-Si substrate. $^{2}$

Indentation was performed on $\sim 920 \mathrm{~nm}$ thick unrelaxed and relaxed $a$-Si layers using an $\sim 5.0 \mu \mathrm{m}$ indenter and loading up to $50 \mathrm{mN}$. The mechanical response of those samples is shown in Fig. 2. The plots show the load versus penetration depth with an inset of the derivative of the load versus penetration depth for both samples. In similar load-unload curves taken for $c$-Si (not shown here) two discontinuities can be observed. ${ }^{5,6,11,13}$ The so-called "pop-in" event during loading and the so-called "pop-out" event during unloading. A pop-in event is related to the transformation of Si-I to the high density metallic Si-II phase during loading, and a popout event is thought to be a result of the transformation of $\mathrm{Si}$-II to the less dense crystalline $\mathrm{Si}-\mathrm{XII} / \mathrm{Si}$-III phases during unloading. ${ }^{11-13,24}$ As reported previously ${ }^{18}$ the load-unload curve of an indent in unrelaxed $a$-Si does not display such discontinuities, as shown in Fig. 2(a). This strongly supports a conclusion that no phase transformations have taken place during both loading and unloading in unrelaxed $a$-Si. However, in the case of relaxed $a$-Si, discontinuities that indicate phase transformations can be observed, as shown in Fig. 2(b). While the pop-out event is clearly visible in the loadunload curve, the pop-in event is less prominent but is clearly evident (a change in slope indicated by an arrow) in the derivative plot. This is similar to the indentation behavior of $c-\mathrm{Si}$ and indicates that a phase transformation from $a-\mathrm{Si}$ to a more dense metallic (possibly Si-II) phase during loading and further phase transformations from that metallic phase to the crystalline phases $\mathrm{Si}-\mathrm{XII}$ and $\mathrm{Si}$-III occur during unloading.

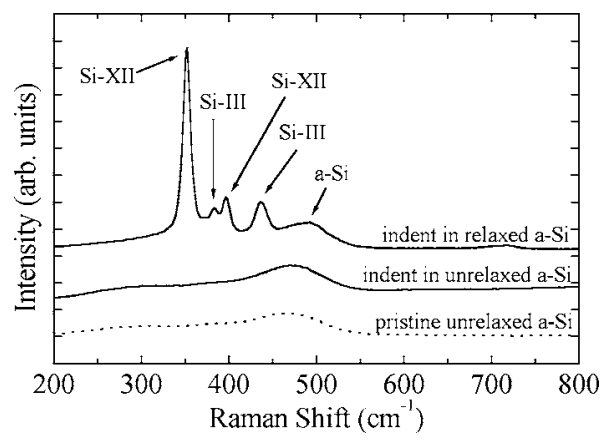

FIG. 3. Raman spectra of pristine $a$-Si and residual indents in unrelaxed and relaxed $\sim 650 \mathrm{~nm}$ thick $a$-Si. Each spectrum is offset for clarity. Note that the spectrum of pristine relaxed $a$-Si is essentially identical to that of unrelaxed $a$-Si. The indentation was performed using an $\sim 5.0 \mu \mathrm{m}$ indenter applying loads up to $50 \mathrm{mN}$.

The Raman spectra from residual indents in $\sim 650 \mathrm{~nm}$ thick unrelaxed and relaxed $a-\mathrm{Si}$ are shown in Fig. 3. The spectrum of pristine, unrelaxed $a$-Si displays the broad $a-\mathrm{Si}$ peak at $470 \mathrm{~cm}^{-1}$, but no $\mathrm{Si}-\mathrm{I}$ peaks, which indicates that the underlying $c$-Si is not sampled by the laser for $a$-Si films of $\sim 650 \mathrm{~nm}$ and thicker. The Raman spectrum from the residual indent in the unrelaxed sample is the same as the spectrum of the unindented $a-\mathrm{Si}$, displaying only the broad $a$-Si peak. This supports the fact that no phase transformations to crystalline phases have taken place in the unrelaxed $a$-Si. In contrast, for the case of $c$-Si the Raman bands of Si-XII (351 and $396 \mathrm{~cm}^{-1}$ ) and Si-III $\left(383\right.$ and $\left.435 \mathrm{~cm}^{-1}\right)$ are found in the spectra of residual indent impressions. ${ }^{11,25}$ These Raman bands are also found in the residual indent impression in relaxed $a$-Si. This confirms that relaxed $a-\mathrm{Si}$ behaves in a very similar manner to $c$-Si in terms of transforming to the $\mathrm{Si}-\mathrm{XII} / \mathrm{Si}$-III phases during unloading.

The occurrence of phase transformations was further investigated by XTEM of residual indents in $\sim 650 \mathrm{~nm}$ thick unrelaxed and relaxed $a$-Si carried out using an $\sim 2.0 \mu \mathrm{m}$ indenter applying loads up to $20 \mathrm{mN}$. These particular XTEM results have been reported previously ${ }^{18}$ but we reshow them here for completeness and to aid in the interpretation and discussion of the thin film results presented in the next section. Consistent with the load-unload curves and the Raman data there is no evidence of transformation in the case of unrelaxed $a$-Si (at a maximum load of $20 \mathrm{mN}$ for an $\sim 2.0 \mu \mathrm{m}$ indenter) in the XTEM micrograph in Fig. 4(a), whereas the dark-field XTEM micrograph in Fig. 4(b) clearly shows the presence of crystalline phases ( $\mathrm{Si}-\mathrm{III}$ and $\mathrm{Si}-\mathrm{XII}$ ) under the residual indent. ${ }^{18}$ Note that there is no evidence of plastic deformation (e.g., slip) or phase transformation in the underlying $c$-Si in either case. This indicates that, for $\sim 650 \mathrm{~nm}$ thick $a$-Si films under the indentation conditions used, deformation is confined to the amorphous films.

\section{B. Thin a-Si films}

Indentation was made on amorphous films of $\sim 100 \mathrm{~nm}$ thickness. In this case the deformation of the underlying $c$-Si is expected to occur at the maximum load conditions since the maximum tip penetration depth exceeds the original film thickness. The indentation behavior of $\sim 100 \mathrm{~nm}$ 

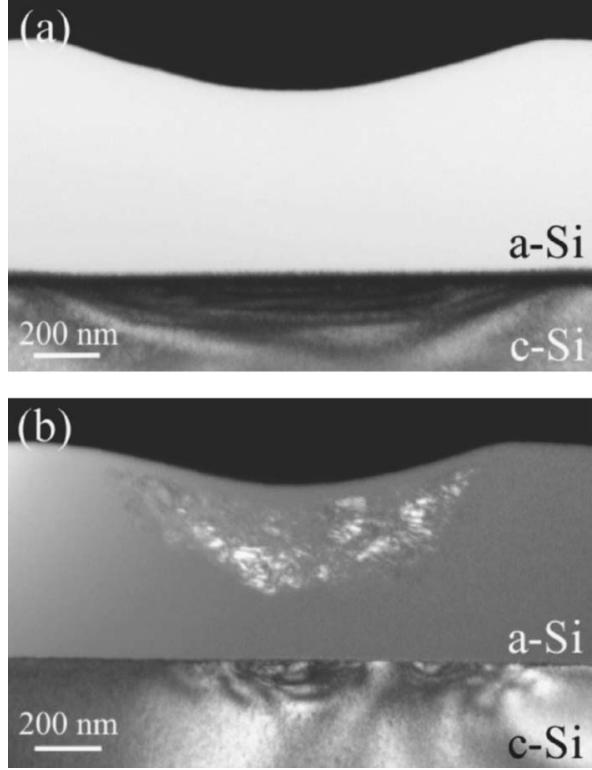

FIG. 4. (a) Bright-field XTEM image of a residual indent in $\sim 650 \mathrm{~nm}$ thick, unrelaxed amorphous silicon. (b) Dark-field XTEM image of a residual indent in $\sim 650 \mathrm{~nm}$ thick, relaxed amorphous silicon. Although strain is clearly present in the underlying $c$-Si, no plastic deformation takes place. The indentation was made using an $\sim 2.0 \mu \mathrm{m}$ indenter applying loads up to $20 \mathrm{mN}$, as shown previously (see Ref. 18).

thick unrelaxed and relaxed $a$-Si is shown in Fig. 5(a). The load-unload curves of both the unrelaxed (shown as an inset) and relaxed cases, are quite similar to $c$-Si, ${ }^{11-13,24}$ with a pop-in event and a pop-out event visible, suggesting that phase transformations occur in both cases.
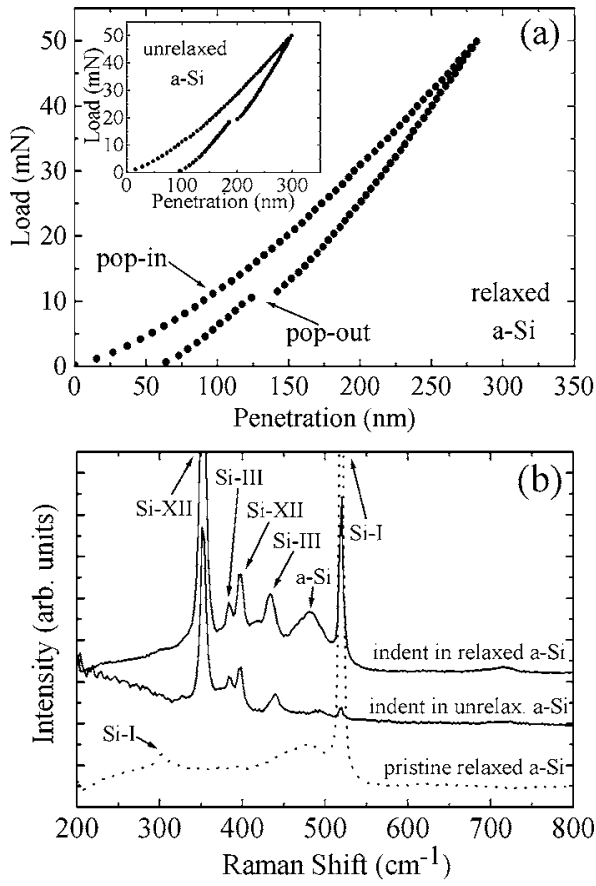

FIG. 5. (a) Load-displacement curves of indentation into relaxed and unrelaxed (shown as an inset) $\sim 100 \mathrm{~nm}$ thick $a$-Si. In both cases a "pop-out" event and a "pop-in" event are visible. (b) Raman spectra of pristine $a$-Si and residual indents in unrelaxed and relaxed $\sim 100 \mathrm{~nm}$ thick $a$-Si. Each spectrum is offset for clarity. Note that the spectrum of pristine relaxed $a-\mathrm{Si}$ is essentially identical to that of unrelaxed $a$-Si. The indentation was performed using an $\sim 5.0 \mu \mathrm{m}$ indenter applying loads up to $50 \mathrm{mN}$ in all cases.


FIG. 6. (a) Load-unload curve of an indent in relaxed $\sim 100 \mathrm{~nm}$ thick $a$-Si. (b) Corresponding dark-field image with inset of the diffraction pattern of a residual indent in $\sim 100 \mathrm{~nm}$ thick relaxed $a$-Si. The reflection used for the dark-field image is marked by a box, with the marked regions showing (1) amorphous layer, [(2) and (3)] transformed regions, and (4) slip. The indentation was performed using the $\sim 2.0 \mu \mathrm{m}$ indenter applying loads up to $20 \mathrm{mN}$.

In addition, the Raman spectra of the residual indents in these films also look quite similar to the spectrum of $c-\mathrm{Si}^{5,11,12,24}$ as shown in Fig. 5(b). For $\sim 100 \mathrm{~nm}$ thick films, the Raman band of $a-\mathrm{Si}\left(470 \mathrm{~cm}^{-1}\right)$ is observed and those of the underlying Si-I $\left(300\right.$ and $520 \mathrm{~cm}^{-1}$ ). Spectra from residual indents in both unrelaxed and relaxed $a$-Si also exhibit Raman bands of Si-XII and $\mathrm{Si}-\mathrm{III}$ as well as those of the surrounding amorphous silicon and the underlying Si-I.

For such thin films neither the load-unload curves nor the Raman spectra can distinguish between phase transformations in the $a$-Si layer and those in the underlying $c$-Si; XTEM of residual indents in $\sim 100 \mathrm{~nm}$ thick $a$-Si was conducted. The indentation was performed using the $\sim 2.0 \mu \mathrm{m}$ indenter applying loads up to $20 \mathrm{mN}$ as shown in Fig. 6(a). The dark-field micrograph of the residual indent in relaxed $a-\mathrm{Si}$ (the amorphous layer is marked by arrows numbered 1), shown in Fig. 6(b), illustrates a transformed region both within the amorphous layer (region of interest 2) and also in the underlying crystalline silicon (region of interest 3 ). The thin amorphous layer directly beneath the residual indent is due to implantation damage caused by the FIB during the initial imaging with the gallium beam of the sample prior to depositing the platinum layer. Beneath the transformed regions, slip is observed (region of interest 4). The Si-III/ Si-XII reflection used for the dark-field image is marked by a box in the diffraction pattern. The diffraction pattern displays not only the amorphous rings and Si-III/Si-XII spots, but also reflections indicating Si-I. In contrast, XTEM images of residual indents in thin unrelaxed $a$-Si films indicate that the $a$-Si layers do not appear to transform at these loads. However, this is not the case at high loads as we illustrate later in Sec. III D. 

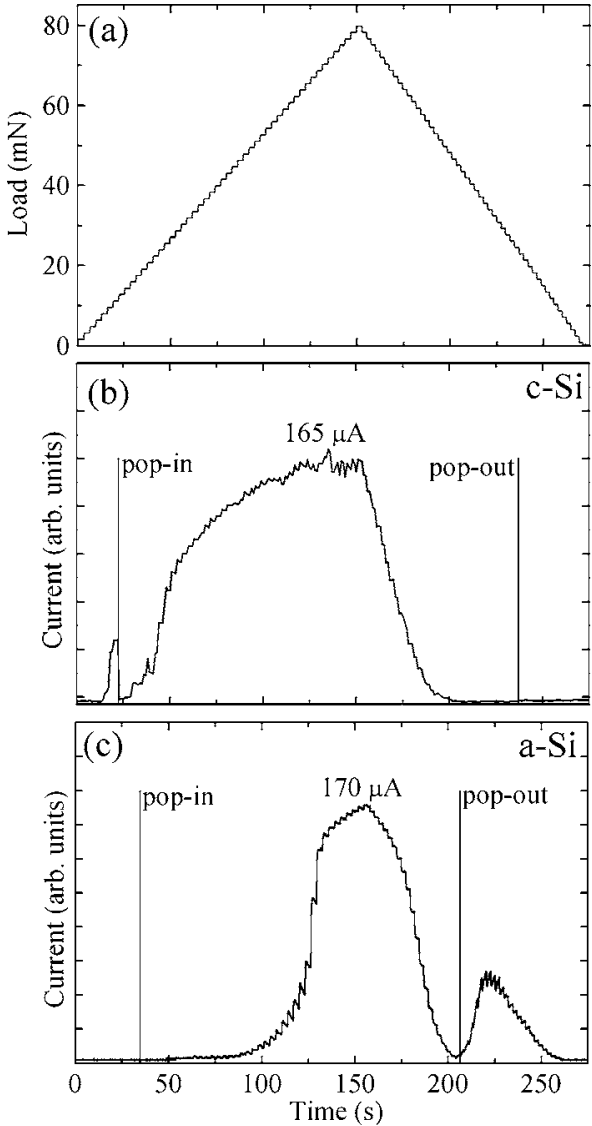

FIG. 7. Electrical measurements taken during the indentation of $c$-Si and $\sim 350 \mathrm{~nm}$ thick relaxed $a$-Si. The indentation load vs time is displayed in (a), while the measured current through the sample vs time for $c$-Si and $a$-Si is shown in (b) and (c), respectively. The "pop-in" and "pop-out" events are marked with a line in each case. The indentation was performed using an $\sim 5.0 \mu \mathrm{m}$ indenter applying loads up to $80 \mathrm{mN}$.

\section{In situ electrical measurements of relaxed a-Si films}

In situ electrical measurements were performed on selected samples. An example of the current flow through the $\mathrm{Al} / \mathrm{Si}$ structure versus time (for $7 \mu \mathrm{m}$ epilayer samples) during indentation is shown in Fig. 7. In this case the electrical behavior was measured under indentation with a spherical indenter of $\sim 5.0 \mu \mathrm{m}$ radius with loads up to $80 \mathrm{mN}$ for a crystalline sample [Fig. 7(b)] and for an $\sim 350 \mathrm{~nm}$ thick relaxed $a$-Si sample [Fig. 7(c)]. Note that the load-unload curves for the $c$-Si and the relaxed $a$-Si show essentially the same behavior as that in Fig. 2(b); only the maximum penetration depth is increased due to the higher load applied. The pop-in and pop-out events, as determined from loadunload curves, are marked in the current versus time plots. This shows that, for $c-\mathrm{Si}$, the current increases significantly even before the pop-in event, behavior we have observed previously. ${ }^{7}$ However, in the case of the relaxed $a-\mathrm{Si}$, no significant current flows until the load reaches $\sim 45 \mathrm{mN}$. Similar to previous reports ${ }^{7}$ the current drops rapidly during unloading. For the case of $a$-Si in Fig. 7(c), an increase in current can be observed during unloading at the pop-out event. Possible reasons for this will be discussed later.

Further electrical measurements were made on relaxed $a$-Si films (created in the $\sim 2 \mu \mathrm{m}$ epilayer) of different thick-

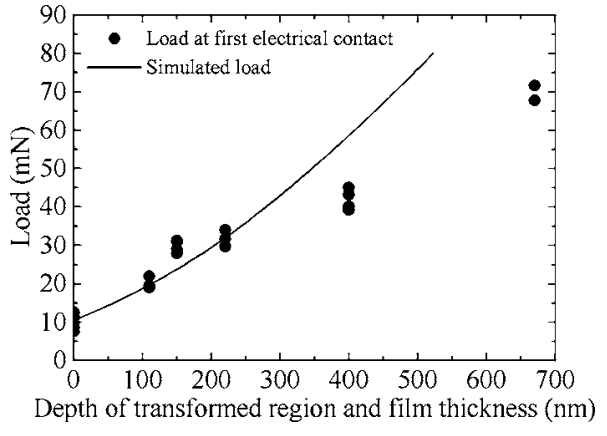

FIG. 8. Applied load at the first electrical contact to the underlying $c$-Si vs the thickness of the film of the respective sample. The indentation was performed using an $\sim 5.0 \mu \mathrm{m}$ indenter applying loads up to $50 \mathrm{mN}$ and up to $80 \mathrm{mN}$. The load-depth dependence of the interface between the transformed and the untransformed region was simulated using ELASTICA (see Ref. 26).

nesses $(\sim 110, \sim 150, \sim 220, \sim 400$, and $\sim 670 \mathrm{~nm})$ with a spherical indenter of $\sim 5.0 \mu \mathrm{m}$ radius applying loads up to $80 \mathrm{mN}$. Figure 8 displays the load at which significant current ( $>5 \%$ increase of current within $1 \mathrm{~s}$ ) flows versus the $a$-Si layer thickness. Interestingly, this load increases roughly linearly with the film thickness. The load-depth dependence of the critical isostatic stress $(\sim 11 \mathrm{GPa})$ necessary for the $c$-Si to Si-II phase transformation was modeled by the computer package ELASTICA (Ref. 26) and this simulation is depicted by the solid line in Fig. 8 .

\section{Unrelaxed a-Si films at high loads}

As previously reported, ${ }^{18}$ the unrelaxed $a$-Si films do not transform under low loads, however, it is possible to induce phase transformations at higher loads. For example, further indentation was performed on an $\sim 650 \mathrm{~nm}$ thick unrelaxed $a$-Si film on $c$-Si by applying a maximum load of $40 \mathrm{mN}$ using the $\sim 2.0 \mu \mathrm{m}$ indenter, resulting in a maximum penetration depth of $\sim 530 \mathrm{~nm}$. In contrast to the behavior of the sample at low loads [see Fig. 2(a)], the load-unload curve, shown in Fig. 9(a), displays a very clear pop-in event during loading and a clear pop-out event during unloading, suggesting phase transformations. The pop-in event takes place at a considerably higher load $(\sim 35 \mathrm{mN}$, corresponding to a penetration depth of $\sim 400 \mathrm{~nm}$ ) and is more prominent than in the cases of $c$-Si and relaxed $a$-Si [see Fig. 2(b)].

XTEM establishes whether a phase transformation actually occurs in the unrelaxed $a-\mathrm{Si}$ or is confined to the underlying $c$-Si in such high load cases. Figure 9(b) shows a XTEM image of such a residual indent. Here the crystalline phases are clearly observed in the unrelaxed $a$-Si film. This result confirms that it is possible for unrelaxed $a$-Si to transform at high loads under a spherical indenter following plastic flow at lower loads. Thus, we interpret the very prominent pop-in event observed in Fig. 9(a) to indicate the sudden onset of a transformation in unrelaxed $a$-Si in material that cannot flow due to confinement between the indenter and the underlying $c$-Si substrate.

The occurrence of phase transformations was further indicated by electrical measurements on an $\sim 350 \mathrm{~nm}$ thick unrelaxed $a$-Si sample (created in an $\sim 7 \mu \mathrm{m} c$-Si epilayer). 

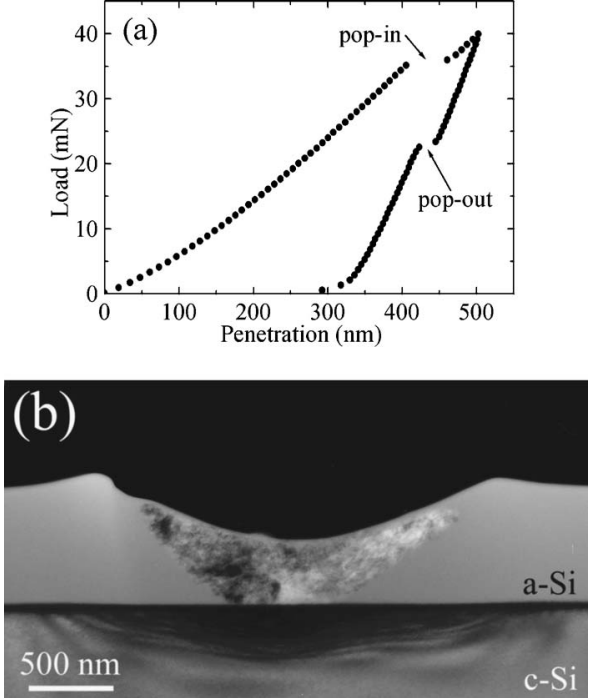

FIG. 9. Load-unload curve (a) and bright-field XTEM image (b) of a residual indent in $\sim 650 \mathrm{~nm}$ thick, unrelaxed $a$-Si. The indentation was performed by using an $\sim 2.0 \mu \mathrm{m}$ indenter applying loads up to $40 \mathrm{mN}$.

Indentations were performed in this case using an $\sim 5.0 \mu \mathrm{m}$ indenter loading up to $80 \mathrm{mN}$ resulting in a maximum penetration depth of $\sim 460 \mathrm{~nm}$, as shown in Fig. 10(a). Again the pop-in event occurs at a higher load $(\sim 35 \mathrm{mN}$, corresponding to a penetration depth of $\sim 350 \mathrm{~nm}$ ) than in the case of the relaxed $a$-Si film [less than $15 \mathrm{mN}$ in Fig. 2(b)]. The electrical behavior of this sample during indentation is shown in Fig. 10(b). Current starts to flow at a load of $\sim 70 \mathrm{mN}$, around $25 \mathrm{mN}$ higher than in the case of a relaxed $a$-Si sample of the same thickness [see Fig. 7(c)]. This again shows that at high loads the unrelaxed $a$-Si can be forced to transform to a metallic phase during loading and then trans-
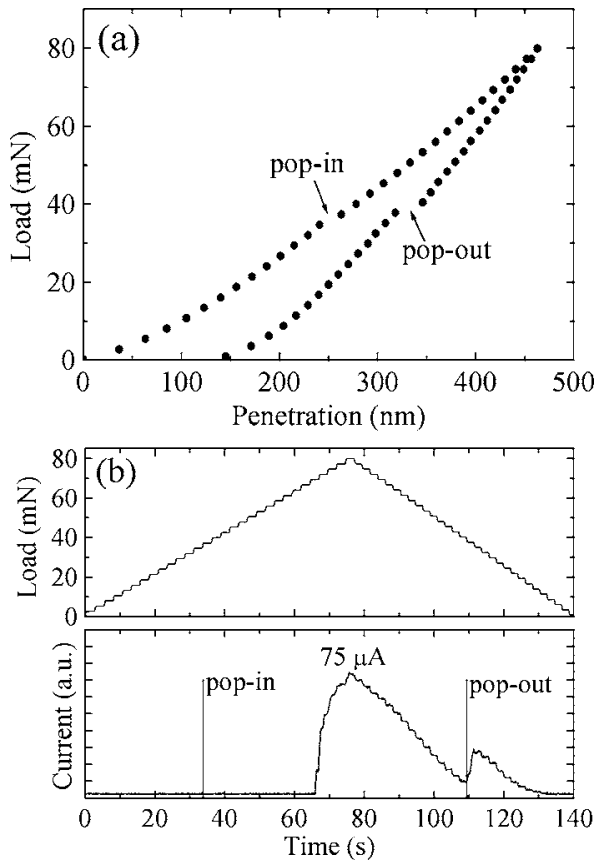

FIG. 10. Load-unload curve (a) and electrical measurement (b) of an indent in $~ 350 \mathrm{~nm}$ thick unrelaxed $a$-Si. The "pop-in" and "pop-out" events are both marked with a line. The indentation was made using an $\sim 5.0 \mu \mathrm{m}$ indenter applying loads up to $80 \mathrm{mN}$. relaxed

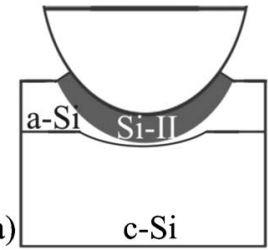

unrelaxed

(b)
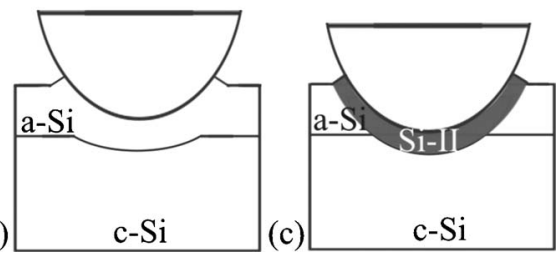

FIG. 11. Schematics of the deformation behavior of relaxed and unrelaxed $a$-Si films: the compression and deformation of a (a) relaxed $a$-Si film, (b) unrelaxed $a$-Si film under low stress conditions and (c) unrelaxed $a$-Si film under high stress conditions. While the unrelaxed $a$-Si film under low stress conditions deforms via plastic flow, the relaxed $a$-Si film and the unrelaxed $a$-Si film under high stress conditions deform by phase transformation.

form further to the crystalline phases during unloading. A sudden increase in the current can be observed corresponding to the pop-out event, behavior that is discussed later.

\section{DISCUSSION}

The results of this study clearly show that there are differences between the mechanical deformation behavior of unrelaxed and relaxed $a$-Si films. All relaxed $a$-Si films undergo plastic deformation in a similar manner to $c$-Si by transforming to a metallic phase during loading and further to the crystalline $\mathrm{Si}-\mathrm{XII} / \mathrm{Si}$-III phases during unloading. Hence, simple mechanical deformation at room temperature can induce a phase transformation from relaxed (low density) $a$-Si to crystalline phases following unloading, independent of the amorphous film thickness. This behavior is not entirely consistent with previous diamond anvil ${ }^{14,15}$ and one other indentation ${ }^{16}$ study, that indicate that, thermodynamically, low density $a$-Si should transform to high density metallic $a-\mathrm{Si}$ on loading and to low density $a-\mathrm{Si}$ again on unloading. Our observations for the indentation of relaxed $a-\mathrm{Si}$, whereby $\mathrm{Si}$-III/Si-XII phases are obtained on unloading, raise the question as to what metallic phase (crystalline Si-II or high density $a-\mathrm{Si}$ ) is present upon loading in our case. Further work is clearly needed to investigate this phenomenon.

Compared with relaxed $a$-Si layers, the deformation behavior of the unrelaxed films is more complex, since both the isostatic stress under the indenter and the confinement of material between indenter and underlying $c$-Si play a critical role in determining the modes of deformation. Before discussing the transformation behavior of these unrelaxed $a-\mathrm{Si}$ films in detail we will first consider the general tip penetration and deformation behavior (both elastic and plastic) of an $a$-Si layer on $c$-Si under increasing load.

Figure 11(a) shows a schematic diagram of a relaxed $a-\mathrm{Si} / c-\mathrm{Si}$ structure during loading. During loading the $a-\mathrm{Si}$ 
layer becomes compressed and starts to transform to a metallic phase under the indenter. If the film is unrelaxed it will begin to deform via the plastic flow of material [see Fig. 11(b)]. Thus, in both cases of relaxed and unrelaxed $a-\mathrm{Si}$ films some material can flow out from under the indenter during loading (the metallic phase in the case of relaxed $a$-Si and $a$-Si itself in the case of the unrelaxed phase). For thick $a$-Si films at such stress conditions, the role of the underlying substrate is less significant. However, when the penetration depth of the tip approaches or exceeds the film thickness, both film and substrate deformation processes are important. In the case of unrelaxed $a$-Si films under such loading conditions [see Fig. 11(c)], the confinement of the film between the indenter and underlying $c$-Si can lead to the phase transformation of the unrelaxed film. Indeed, the deformation of unrelaxed $a-\mathrm{Si}$ films is a function of stress (isostatic stress under the indenter and confinement between indenter and substrate). The applied maximum load does not significantly influence the deformation behavior of thin unrelaxed $a$-Si films. At either high or low loads the thin $a$-Si layer under the indenter appears to flow out plastically from under the tip. The isostatic stress in this case is not high enough to induce any phase transformation because of the lack of confinement. The underlying $c$-Si deforms where, depending on the loading conditions, slip and phase transformations can be induced. In the case of thick unrelaxed $a-\mathrm{Si}$ layers, where, under our maximum loading conditions, the tip penetrates to less than one-half of the layer thickness, the unrelaxed $a$-Si also flows out plastically to relieve the stress and no phase transformations are observed. However, the deformation mode differs at intermediate thickness and load regime, when at maximum load the tip penetrates to a depth roughly equal to the film thickness. In this case phase transformations can be observed (see Fig. 9) implying that, when some (enough) unrelaxed material is confined under the indenter and pressed against the underlying $c$-Si, this constraint forces the material to transform in a similar way to relaxed $a$-Si as the stress increases. Thus, although the preferred transformation mode of unrelaxed $a$-Si is plastic flow, it can transform if the stress conditions are appropriate.

We now focus on the in situ electrical measurement results to assist in better understanding the deformation and phase transformation behavior of the films. We first consider the electrical behavior during the loading cycle. In the case of $c$-Si, the onset of the phase transformation to $\mathrm{Si}$-II directly under the indenter converts a portion of the Schottky contact to an Ohmic contact via the formation of an (intermediate) metallic Si-II layer. ${ }^{7,27,28}$ The pop-in event is often accompanied by a sudden drop in the current [see Fig. 7(b)], which we have previously ${ }^{7}$ interpreted as metallic Si-II flowing out from under the indenter and transforming to (insulating) $a$-Si. Thereafter, the current increases with the increased contact area between transformed (Si-II) material and the $\mathrm{Al}$ layer. In the case of the relaxed $a$-Si films a different electrical behavior is observed, since no significant current can flow until the transformed metallic phase penetrates through the insulating $a$-Si to the underlying $c$-Si. After electrical connection is made with the underlying $c$-Si the current continues to increase with increasing contact area as in the case of $c$-Si. The similar maximum current flowing at maximum load that we observe might indicate that the same phase is formed at loading for $c$-Si and relaxed $a$-Si. A similar behavior is observed in the case of the unrelaxed $a$-Si if subjected to loading conditions that induce a phase transformation in the $a$-Si layer. The huge increase in current at its onset is due to the sudden transformation of the whole volume of material confined between the indenter and the substrate, which is indicated by the large pop-in event.

Let us now consider the $a$-Si film thickness dependence on the onset of current flow for relaxed films. The load at which this occurs increases roughly linearly with the film thickness. Understanding this behavior is nontrivial since details of the elastic and plastic deformation of the $a$-Si films need to be considered as well as the volume of transformed metallic phase under the tip and that which is "lost" by plastic flow. The prediction of the onset of current flow is not possible by comparison with the thickness of transformed material estimated from ELASTICA (Ref. 26) simulations as it assumes that the material below the indenter has the mechanical properties of $c-\mathrm{Si}$ and only accounts for the elastic compression of the material and the penetration of the tip. Nevertheless, even with such a simple model there is good agreement for the thinner films, between the depth estimated for the extent of Si-II and the onset of the current flow.

We now turn our attention to the more complex unloading behavior. For the case of $c$-Si the current drops rapidly to the preloading value after unloading begins. We previously suggested that this resulted from a transformation of $\mathrm{Si}$-II to much higher resistivity $\mathrm{Si}-\mathrm{III} / \mathrm{Si}$-XII or $a$-Si phases on unloading. ${ }^{7}$ Even a thin layer of high resistance phase between the $\mathrm{Si}$-II volume and $c$-Si would cause the current to drop rapidly. With a different setup for the electrical measurement (for example, placing the sample under forward bias) a slight increase in current can be observed to accompany a pop-out event despite this large current drop. Some earlier electrical measurements ${ }^{28}$ also showed evidence for such a current increase on unloading and attributed this to cracking. However, there is no evidence of cracking under our experimental conditions. Other electrical measurements ${ }^{29,30}$ attributed this sudden increase in conductivity to a sudden transformation of the Si-XII/Si-III phases to a pressurised (metallic) $a-\mathrm{Si}$, which transforms further to insulating $a-\mathrm{Si}$ upon final unloading. However, there is no evidence of cracking under our experimental conditions and the $\mathrm{Si}-\mathrm{XII} / \mathrm{Si}$-III phases are clearly present in residual indents performed with such slow unloading and relatively little loads. Intriguingly, in the case of $a$-Si the increase in current on unloading is more apparent [see Figs. 7(c) and 10] and this behavior again coincides with the pop-out event. One interpretation of this sudden increase is that the resistivity of some of the material under the indenter decreases.

Since we do not fully understand the origin of the increase in current on unloading we are now undertaking a series of experiments, including more sensitive electrical measurements using a conducting diamond tip, the results of which will be published in a future article. To explain this increase in current we can offer the following scenarios. The first possibility is an experimental artifact whereby the pop- 
out event accompanies a sudden increase in material volume. If our system cannot respond fast enough to keep the load at pop out constant, a transformation back to Si-II of some of the $\mathrm{Si}$-III/Si-XII transformed material may lead to a corresponding current increase. Although we cannot completely rule out this possible cause, the current transient appears to be much longer $(\sim 50 \mathrm{~s})$ than the expected response time of our ultra-micro-indentation-system (UMIS) and we therefore favor material-based explanations such as the following. The pop-out event is thought to be caused by a sudden onset of a transformation of a volume of $\mathrm{Si}$-II to the less dense $\mathrm{Si}$-III and Si-XII phases. The question arises as to what the resistivity of these latter phases would be. While Si-III is a semimetal with a resistivity of less than $0.1 \Omega \mathrm{cm},{ }^{31,32}$ there is some evidence that $\mathrm{Si}-\mathrm{XII}$ is a semiconductor with a narrow band gap rather than a semimetal. ${ }^{33}$ Hence it may be possible that the metallic Si-II first transforms to the semiconducting (higher resistivity) Si-XII phase at the Si-II/Si-I interface and then much of this phase transforms to the semimetallic (low resistivity) Si-III at pop out. This would decrease, once more, the resistivity of the region beneath the indenter and hence increase the measured current. However, such a transformation does not explain the pop-out event which has been widely interpreted as the sudden nucleation of the $\mathrm{Si}-\mathrm{XII} / \mathrm{Si}$-III phases and hence a large change in volume, noting that Si-III and Si-XII have comparable densities. ${ }^{9}$

An alternative explanation is that, on unloading, metallic $\mathrm{Si}$-II or high density $a$-Si first transforms to a lower density $a$-Si phase that grows in thickness from the Si-II/Si-I interface. This amorphous phase would be expected to be less dense than $\mathrm{Si}$-II and metallic $a$-Si but more dense than $\mathrm{Si}$-III and Si-XII. Thus, if such a relatively high density $a$-Si phase exists during unloading it would need to be of high resistivity and also then transform into the less dense Si-III and $\mathrm{Si}$-XII phases (that have lower resistivity than $a$-Si) at pop out. This last explanation would be consistent with a current increase at pop out but relies on the formation of a highly resistive, high density $a$-Si phase on initial unloading. Such a phase is yet to be identified, but a recent paper $^{34}$ may provide partial evidence for its existence. In this report McMillan et al. proposed that high density (metallic) $a$-Si can transform to low density (insulating) $a$-Si over a range of pressures. They indicate that mixed $a$-Si phases may exist over a wide pressure range $(8-4 \mathrm{GPa})$. If this is the case then a nanostructured mixture of metallic (high density) $a$-Si and insulating (low density) $a$-Si may provide an average density lower than Si-II and an effective high resistivity. The resistivity may be expected to decrease (i.e., giving an increase in current) at pop out when $\mathrm{Si}-\mathrm{XII} / \mathrm{Si}$-III phases form from the high density $a$-Si in the mixture.

\section{CONCLUSIONS}

The occurrence of deformation and phase transformations in both relaxed and unrelaxed ion-implanted surface $a$-Si films of different thicknesses has been studied. Ex situ measurements such as Raman microspectroscopy and XTEM have been used, which reveal the final structural states of the region beneath residual indent impressions. Independent of the film thickness, relaxed $a$-Si seems to transform similarly to $c$-Si, with a transformation to a metallic $\mathrm{Si}-\mathrm{II}$ or high density $a$-Si phase during loading and further transformation to the crystalline phases $\mathrm{Si}-\mathrm{XII}$ and $\mathrm{Si}-\mathrm{III}$ or to $a$-Si during unloading. While the preferred transformation mode of unrelaxed $a$-Si is plastic flow, under certain stress conditions it can be forced to transform in a similar way to relaxed $a$-Si. The Si epilayer structure used has enabled an in situ electrical study of the transformation behavior of these $a$-Si films. The electrical response to a metallic phase transformation is similar with $a-\mathrm{Si}$ and to previously observed behavior in $c$-Si, but significant current can only flow in the $a$-Si case once the transformed metallic phase extends through the insulating $a$-Si layer to connect to the underlying $c$-Si. Simulations of the expected load at which the onset of current should occur are in good agreement for thin films but depart for thicker $a$-Si films. During unloading a second increase in current has been observed at "pop out," which cannot be fully explained yet. We have suggested that this behavior is a result of a phase transformation at pop out, to a more conducting phase, but this needs to be validated by further experiments.

\section{ACKNOWLEDGMENT}

The authors would like to thank M. V. Swain from the University of Otago for the helpful discussions.

${ }^{1}$ D. E. Carlson and C. R. Wronski, in Amorphous Semiconductors, edited by M. H. Brodsky (Springer-Verlag, Berlin, 1979), pp. 287-329.

${ }^{2}$ D. M. Follstaedt, J. A. Knapp, and S. M. Myers, J. Mater. Res. 19, 338 (2004).

${ }^{3}$ J. Williams, Y. Chen, J. Wong-Leung, A. Kerr, and M. Swain, J. Mater. Res. 14, 2338 (1999).

${ }^{4}$ J. Z. Hu, L. D. Merkle, C. S. Menoni, and I. L. Spain, Phys. Rev. B 34, 4679 (1986).

${ }^{5}$ A. Kailer, Y. G. Gogotsi, and K. G. Nickel, J. Appl. Phys. 81, 3057 (1997).

${ }^{6}$ Y. Gogotsi, V. Domnich, S. N. Dub, A. Kailer, and K. Nickel, J. Mater. Res. 15, 871 (2000).

${ }^{7}$ J. E. Bradby, J. S. Williams, and M. V. Swain, Phys. Rev. B 67, 085205 (2003).

${ }^{8}$ J. Crain, G. J. Ackland, J. R. Maclean, R. O. Piltz, P. D. Hatton, and G. S. Pawley, Phys. Rev. B 50, 13043 (1994).

${ }^{9}$ R. O. Piltz, J. R. Maclean, S. J. Clark, G. J. Auckland, P. D. Hatton, and J. Crain, Phys. Rev. B 52, 4072 (1995).

${ }^{10}$ M. Imai, T. Mitamura, K. Yaoita, and K. Tsuji, High Press. Res. 15, 167 (1996).

${ }^{11}$ J. E. Bradby, J. S. Williams, J. Wong-Leung, M. V. Swain, and P. Munroe, J. Mater. Res. 16, 1500 (2001).

${ }^{12}$ V. Domnich, Y. Gogotsi, and S. Dub, Appl. Phys. Lett. 76, 2214 (2000).

${ }^{13}$ J. E. Bradby, J. S. Williams, J. Wong-Leung, M. V. Swain, and P. Munroe, Appl. Phys. Lett. 77, 3749 (2000).

${ }^{14}$ O. Shimomura, S. Minomura, N. Sakai, K. Asaumi, K. Tamura, J. Fukushima, and H. Endo, Philos. Mag. 29, 547 (1974).

${ }^{15}$ S. K. Deb, M. Wilding, M. Somayazulu, and P. F. McMillan, Nature (London) 414, 528 (2001).

${ }^{16}$ D. R. Clarke, M. C. Kroll, P. Kirchner, R. F. Cook, and B. J. Hockey, Phys. Rev. Lett. 60, 2156 (1988).

${ }^{17}$ M. M. Khayyat, G. K. Banini, D. G. Hasko, and M. M. Chaudhri, J. Phys. D 36, 1300 (2003).

${ }^{18}$ B. Haberl, J. E. Bradby, M. V. Swain, J. S. Williams, and P. Munroe, Appl. Phys. Lett. 85, 5559 (2004).

${ }^{19}$ S. Roorda, S. Doorn, W. C. Sinke, P. M. L. O. Scholte, and E. van Loenen, Phys. Rev. Lett. 62, 1880 (1989).

${ }^{20}$ S. Roorda et al., Phys. Rev. B 44, 3702 (1991).

${ }^{21}$ J. M. Gibson, J.-Y. Cheng, P. Voyles, M. M. J. Treacy, and J. C. Jacobson, Mater. Res. Soc. Symp. Proc. 540, 27 (1999) 
${ }^{22}$ J.-Y. Cheng, J. M. Gibson, and D. C. Jacobson, J. Mater. Res. 16, 3030 (2001).

${ }^{23}$ G. L. Olson and J. A. Roth, Mater. Sci. Rep. 3, 1 (1988)

${ }^{24}$ T. Juliano, V. Domnich, and Y. Gogotsi, J. Mater. Res. 19, 3099 (2004).

${ }^{25}$ A. Kailer, K. G. Nickel, and Y. G. Gogotsi, J. Raman Spectrosc. 30, 939 (1999).

${ }^{26}$ T. Chudoba and N. Schwarzer, SCISOFT ELASTICA Version 1.01, TU Chemnitz (1999).

${ }^{27}$ I. V. Gridneva, Y. V. Milman, and V. I. Trefilov, Phys. Status Solidi A 14 117 (1972).

${ }^{28}$ G. M. Pharr, W. C. Oliver, R. F. Cook, P. D. Kirchner, M. C. Kroll, T. R. Dinger, and D. R. Clarke, J. Mater. Res. 7, 961 (1992).
${ }^{29}$ A. B. Mann, D. van Heerden, J. B. Pethica, and T. Weihs, J. Mater. Res. 15, 1754 (2000).

${ }^{30}$ A. B. Mann, D. van Heerden, J. B. Pethica, P. Bowes, and T. Weihs, Philos. Mag. A 82, 1921 (2002).

${ }^{31}$ J. M. Besson, E. H. Mokhtari, J. Gonzalez, and G. Weill, Phys. Rev. Lett. 59, 473 (1987).

${ }^{32}$ G. Weill, J. L. Mansot, G. Sagon, C. Carlone, and J. M. Besson, Semicond. Sci. Technol. 4, 280 (1989).

${ }^{33}$ B. G. Pfrommer, M. Côté, S. G. Louie, and M. L. Cohen, Phys. Rev. B 56, 6662 (1997).

${ }^{34}$ P. F. McMillan, M. Wilson, D. Daisenberger, and D. Machon, Nat. Mater. 4, 680 (2005). 